\title{
Photodynamic Therapy for the Treatment of American Tegumentary Leishmaniasis: Evaluation of Therapies Association in Experimentally Infected Mice With Leishmania (Leishmania) amazonensis
}

\author{
Roberta Ayres Ferreira do Nascimento Volpe ${ }^{1}$, Taisa Rocha Navasconi ${ }^{1}$, Vanessa Nesi dos Reis ${ }^{1}$, Noboru \\ Hioka $^{2}$, Tânia Cristina Alexandrino Becker ${ }^{3}$, Maria Valdrinez Campana Lonardoni ${ }^{4}$, Sandra Mara Alessi \\ Aristides $^{4}$, Thaís Gomes Verzignassi Silveira ${ }^{4 *}$
}

${ }^{1}$ Graduate Program in Health Sciences, Universidade Estadual de Maringá, Maringá, Brazil

${ }^{2}$ Department of Chemistry, Universidade Estadual de Maringá, Maringá, Brazil

${ }^{3}$ Departament of of Basic Health Sciences, Universidade Estadual de Maringá, Maringá, Brazil

${ }^{4}$ Department of Clinical Analysis and Biomedicine, Universidade Estadual de Maringá, Maringá, Brazil

\section{*Correspondence to \\ Thaís Gomes Verzignassi Silveira, \\ Universidade Estadual de Maringá Maringá, Brazil. \\ Avenue Colombo, 5790 \\ Zip code: $87020-900$ \\ Tel: +55-4430114878; \\ Fax: +55-4430114860; \\ Email: tgvsilveira@uem.br}

Published online 17 September 2018

\begin{abstract}
Introduction: American tegumentary leishmaniasis (ATL) is a zoonotic disease caused by protozoan parasites of the genus Leishmania that affects the skin and mucous membrane. Currently, the available drugs for the treatment are injectable, with side effects, long-term treatment regimen and there is the possibility of drug resistance. Thus, alternative therapies have been tested, including photodynamic therapy (PDT). We evaluated the efficacy of PDT on its own and associated with the prescribed ATL treatment.

Methods: BALB/c mice were infected with Leishmania (Leishmania) amazonensis and divided into 6 groups: Gluc+PDT, treated with Glucantime ${ }^{\circledR}$ and photodynamic therapy (PDT) with

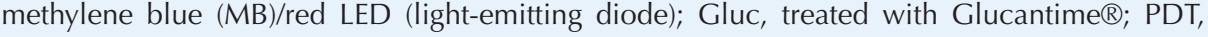
treated with PDT with MB/red LED; Ampho+PDT, treated with amphotericin and PDT with $\mathrm{MB} /$ red LED; Ampho, treated with amphotericin; and control, which were infected but not treated. Two treatment cycles were performed. After 165 days of infection, the parasite load was determined.

Results: Statistical differences were not found $(P>0.05)$ between measures of volume and thickness of the infected footpads in the treated groups when compared with the control group. However, there was a significant reduction $(P<0.05)$ in the parasitic load of the popliteal lymph nodes of the Gluc+PDT, Gluc, PDT and Ampho groups when compared to the control group. In the histological analysis of the infected footpads, the Gluc+PDT group presented a smaller amount of amastigote nests and lower intensity of the mononuclear infiltrate when compared to the Gluc and PDT groups.

Conclusion: The results showed that although there is no significant difference in the evaluations of footpad size (thickness and volume), there is a downward measurement tendency in the Gluc+PDT group, as it can be observed by volume data and corroborated by parasite negative load.

Keywords: Photochemotherapy; Methylene blue; Leishmaniasis, Cutaneous; Leishmania
\end{abstract}

\begin{abstract}
Introduction
Leishmaniasis is an infectious parasitic disease considered by the World Health Organization (WHO) as one of the major world endemics due to its high detection coefficient and high capacity of producing deformities. It is estimated that 12 million people are infected in the whole world and 350 million are at risk of becoming ill. ${ }^{1-3}$

In Brazil, American tegumentary leishmaniasis (ATL) is endemic and represents the second most spread tropical
\end{abstract}

disease. ${ }^{4}$ ATL is an infectious and non-contagious disease, caused by several species of protozoa of the genus Leishmania which affects the skin and mucous membranes, and it clinically appears as cutaneous (CL), mucous cutaneous (MCL) and diffuse cutaneous (DCL). The CL form is the most common, being responsible for more than $90 \%$ cases in Brazil. ${ }^{3}$ It is estimated that $3 \%$ to $5 \%$ of CL cases develop mucosal lesions. ${ }^{4}$

Nowadays, the medicines available in Brazil to treat 
leishmaniasis are injectable, through intramuscular or intravenous administration. The drugs used are pentavalent antimonials (first choice), amphotericin B and pentamidine (second choice). ${ }^{5}$ However, these drugs cause severe side effects, such as cardiotoxicity, pancreatitis, leukopenia, thrombocytopenia, hepatotoxicity and nephrotoxicity, besides requiring prolonged time to eliminate the infection, which, in many cases, causes the abandonment of therapy. ${ }^{6-12}$ In addition to serious adverse effects, reports of the existence of primary and secondary resistance to antimonials may also contribute to the failure of treatment of ATL. ${ }^{13}$

Considering the toxicity, injectable administration, cost and resistance to medicines, added over the long treatment time, alternative forms of therapy have been tested, among these, the photodynamic therapy (PDT). ${ }^{6,14-16}$

PDT is based on the application of a photosensitive substance on the target lesion, which, by receiving the adequate wavelength light, is activated and triggers a series of reactions in the presence of molecular oxygen, resulting in the production of reactive oxygen species, leading to cell death and destruction of diseased tissue. ${ }^{17}$ The simultaneous presence of light, photosensitizing agent and oxygen is fundamental for PDT. ${ }^{17,18}$ Currently, PDT is used in medical areas mainly for the treatment of tumors and some benign dermatological diseases. ${ }^{19}$

Photosensitizers may be dyes, which are photosensitive substances, usually nontoxic for topical application, characterized by a high capacity to absorb visible light. Some of these compounds are capable of reacting photochemically, which turns them into therapeutic agents. ${ }^{18}$ One example is methylene blue $(\mathrm{MB})$, used in medical practice for many years to treat methemoglobinemia, malaria and as urinary antiseptic in the absence of light. MB has bacteriostatic, antiviral and antifungal effects and acts on vascular musculature..$^{20,21}$ Additionally, $\mathrm{MB}$, when excited by red light, produces oxygen "singlet". Still, it presents the advantage of being low cost, easy to acquire and causes little damage to the patient. ${ }^{15}$

Recently, studies have shown the efficiency of PDT in the treatment of CL. ${ }^{6,14,22-24}$ One of the first reports about PDT for CL was made by Gardlo et al, in a study with a 34-year-old man with multiple injuries of CL by Leishmania donovani, in Libya. The patient was treated previously with sodium stibogluconate (Pentostam ${ }^{\circledR}$ ), unsuccessfully, and later the treatment of 5 injuries was done with an association of methyl aminolevulinate (ALA) using red light $\left(75 \mathrm{~J} / \mathrm{cm}^{2}\right)$ and 5 injuries with paromomycin ointment once a day. PDT was performed twice a week for 12 weeks and, later, once a week for 4 more weeks. All injuries treated with PDT obtained clinical and histopathological cure, without relapse after 10 months of after treatment follow-up and excellent cosmetic result. This fact confirms that PDT is a treatment superior to paromomycin, with a complete rate of response $(93 \%$ and
$41 \%$, respectively). ${ }^{19}$

Studies have demonstrated the complete resolution of CL lesion, the decrease in the number of parasites in lesions and leishmanicidal activity to amastigote and promastigote forms. ${ }^{14,24,25}$ The favorable effect of PDT on CL is not only due to the straight parasiticidal effect, but also to the activation of the immune system with liberation of tumor necrosis factor, increase in the expression of adhesion molecules and increase of interleukin $6{ }^{22}$

Among the studies that demonstrated benefit from the use of PDT in the treatment of CL, the majority used a porphyrin precursor (aminolevulinic acid or methylaminolevulinic acid) as photosensitizer agent and laser as light source. ${ }^{24-29}$ As these precursors and light source are high cost and considering the fact that a large number of CL cases occur in underdeveloped countries, dyes such as $\mathrm{MB}$ have been tested as photosensitizing agents and LED as light source. Peloi et al and Sbeghen et al utilized topic MB associated to red LED to treat CL, obtaining a decrease in lesion thickness and parasitic load..$^{16,30}$

As far as our knowledge goes, it has not been evaluated in vivo if PDT using LED as light source and $\mathrm{MB}$ as the photosensitizer in association with reference medicines could contribute to CL treatment. In this study, we evaluated the associations of PDT with LED and MB with Glucantime ${ }^{\circledR}$ or amphotericin $B$ in the treatment of animals experimentally infected with Leishmania amazonensis.

\section{Methods}

\section{Parasites}

Leishmania (Leishmania) amazonensis (MHOM/ BR/1977/LTB0016) was kept in mice by inoculation of $10^{7}$ parasites on the hind footpads. The animals were euthanized after anesthesia using ketamine hydrochloride (160 mg/kg) and xylazine (50 mg/kg), via intraperitoneal. Fragments of regional popliteal lymph nodes from the animals were inoculated aseptically in 199 culture medium (Gibco, New York, USA) $\mathrm{pH} 7.2$ at $25^{\circ} \mathrm{C}$ in a B.O.D. incubator (Logen Scientific, Brazil). Protozoan cultures were kept through weekly subcultures.

\section{Animals}

For the experimental infection 90 days old female $\mathrm{BALB} / \mathrm{c}$ mice were used, obtained from Biotério Central of the State University of Maringá, Maringá, Paraná, Brazil. During the experiment, the animals were kept in the Biotério Setorial at Leishmaniasis Laboratory in mini-insulators in ventilated racks (Alesco, Monte Mor, SP, Brazil) with controlled temperature, bright dark cycles, and environmental barriers. The animals were sedated with a combination of ketamine $(80 \mathrm{mg} / \mathrm{kg})$ and xylazine $(16 \mathrm{mg} / \mathrm{kg})$ before the procedure. At the end of treatment, to obtain biological material, the animals were anesthetized and euthanized. 


\section{Chemicals}

Methylene blue (MB, $\mathrm{C}_{16} \mathrm{H}_{18} \mathrm{C}_{1} \mathrm{~N}_{3} \mathrm{~S}_{3} \mathrm{H}_{2} \mathrm{O}, \mathrm{MW} 373.90 \mathrm{~g}$ $\left.\mathrm{mol}^{-1}\right)$ was prepared at $0.5 \%(\mathrm{~m} / \mathrm{v})$ in aqueous solution. Glucantime $^{\circledR}$ (N-methylglucamine antimoniate, Sanofi Aventis, São Paulo, Brazil) and amphotericin B (Cristália, São Paulo, Brazil) were used as reference treatments.

\section{Lighting System}

Two lighting systems were prepared, having a plate of red LED formed by 6 rows of light-emitting diode (LED) units with $75 \mathrm{~cm}$ total length and $3 \mathrm{~cm}$ width, grasped by a wooden base at the sides of the board, distant $4 \mathrm{~cm}$ (in the first system) and $1 \mathrm{~cm}$ (in the second system) of the LED. Between the LEDs and the animals' footpad a metal screen was put to support the animal. To individualize the treatment, each animal was separated in compartments of $15 \mathrm{~cm}$ using PVC tubes (polyvinyl chloride) with openings of $3 \mathrm{~cm}$ in diameter (Figure 1).

Two lighting systems were utilized with the LED maximum emission at $663 \mathrm{~nm}$ (Table 1).

The emission spectrum of LED was obtained in a spectrofluorometer Varian - Cary Eclipse (Agilent Technologies, USA) and light power was measured by a Portable Laser Power Meter (Edmund Optics Inc., USA). MB absorption spectrum (maximum $665 \mathrm{~nm}$ ) was registered in the spectrophotometer model Varian Cary50.

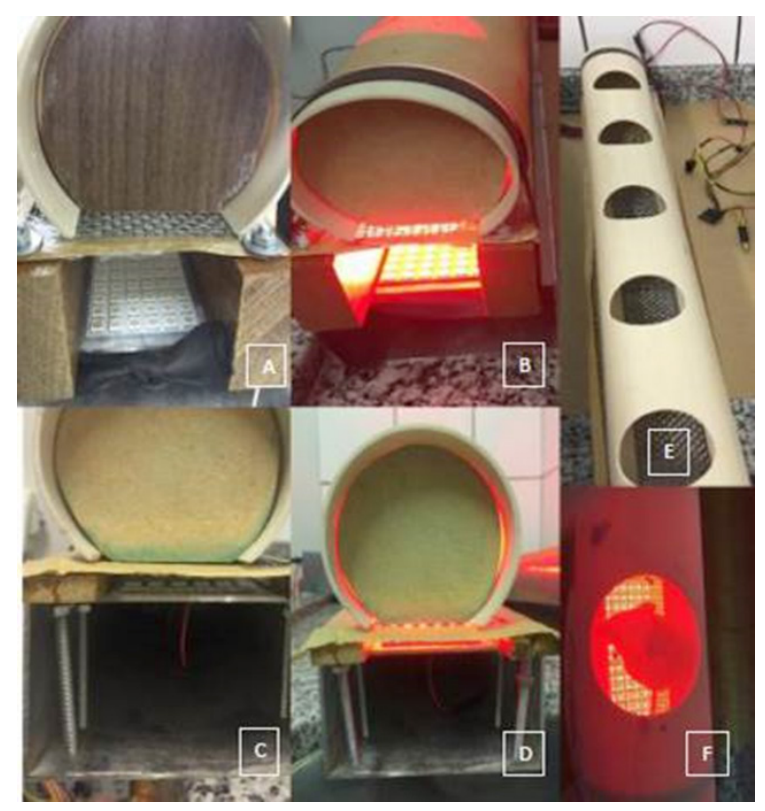

Figure 1. Lighting system consisting of a red LED plate formed by 6 rows of LEDs with a length of $75 \mathrm{~cm}$ and width of $3 \mathrm{~cm}$, secured by a wooden support on the sides of the plate where a metal screen was placed to support the animal: (A) First lighting system with LED plate at distance of $4 \mathrm{~cm}$; (B) First lighting system with LED on; (C) Second lighting system with LED plate at short distance, of $1 \mathrm{~cm}$; (D) Second lighting system with LED on; (E) each animal per individualized compartment $-15 \mathrm{~cm}$ with apertures of $3 \mathrm{~cm}$ in diameter; (F) details showing the individualized compartment with the animals and LED on.
Table 1. Characteristics of Lighting Systems Used

\begin{tabular}{lcc}
\hline Lighting System & $\begin{array}{c}\text { Distance LED Plate } \\
\text { to Animal }\end{array}$ & $\begin{array}{c}\text { Irradiance }\left(\mathbf{1 0}^{\mathbf{3}} \boldsymbol{\mu W a t t} /\right. \\
\left.\mathbf{c m}^{\mathbf{2}}\right)\end{array}$ \\
\hline First & $3 \mathrm{~cm}$ & 2.63 \\
Second & $1 \mathrm{~cm}$ & 4.27 \\
\hline
\end{tabular}

Experimental Infection

The animals were infected with intradermal inoculation containing $5 \times 10^{5}$ promastigote forms in the exponential phase of growth of L. (L.) amazonensis in $100 \mu \mathrm{L}$ saline solution $(0.9 \% \mathrm{NaCl}, \mathrm{m} / \mathrm{v})$ on the plantar pad of the right hind foot. The animals were examined weekly to evaluate the development of lesions (Figure 2).

The animals were divided into 6 groups:

Gluc+PDT group $(\mathrm{n}=5)$ : treated with Glucantime ${ }^{\circledR}(100$ $\mathrm{mg} / \mathrm{kg} / \mathrm{d}=28 \mathrm{mg} \mathrm{Sb} / \mathrm{kg} / \mathrm{d}$ ) via intraperitoneal (IP) during 20 days ( 5 times a week for 4 weeks) and PDT after topic application of $\mathrm{MB}$;

Gluc group $(\mathrm{n}=5)$ : treated with Glucantime ${ }^{\circledR}(100 \mathrm{mg} /$ $\mathrm{kg} / \mathrm{d}=28 \mathrm{mg} \mathrm{Sb} / \mathrm{kg} / \mathrm{d}$ ) via intraperitoneal for 20 days (5 times a week for 4 weeks);

PDT group $(\mathrm{n}=4)$ : treated with PDT after topical application of $\mathrm{MB}$;

Ampho+PDT group $(\mathrm{n}=4)$ : treated with amphotericin $\mathrm{B}(5 \mathrm{mg} / \mathrm{kg} / \mathrm{d})$ via intraperitoneal for 20 days (5 times a week for 4 weeks) and PDT therapy after topic application of $\mathrm{MB}$;

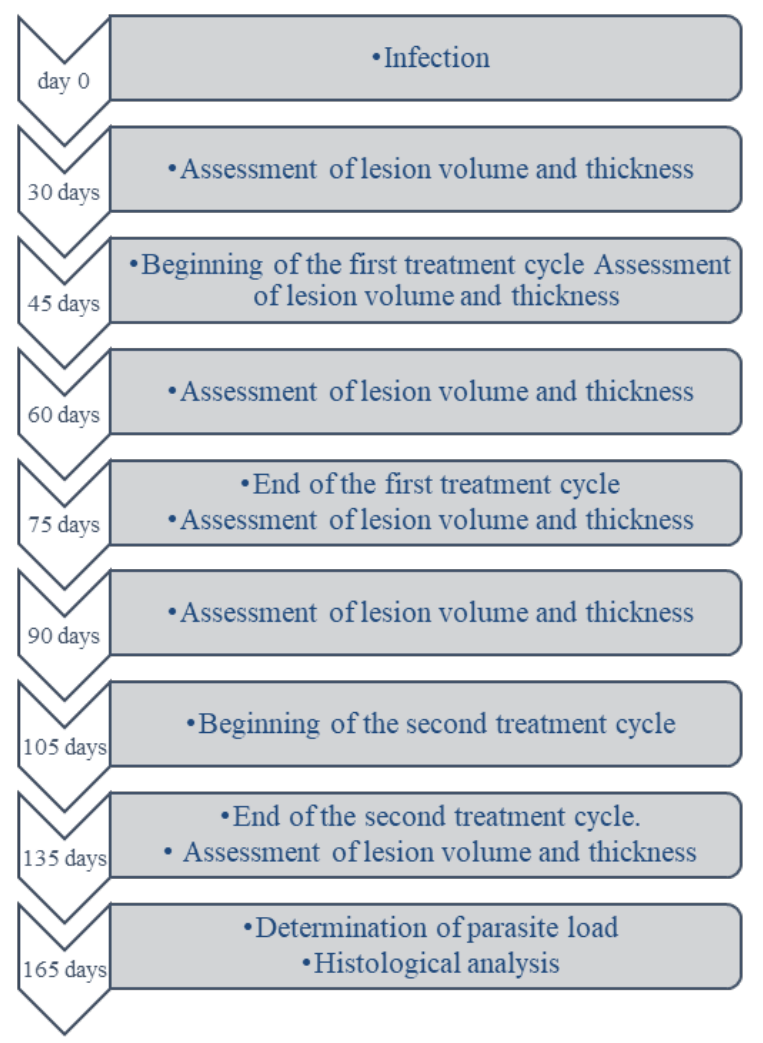

Figure 2. Flowchart of the Experiment. Procedures performed from the infection of the animals (day 0 ) until the determination of the parasite load 165 days after the infection. 
Ampho group $(\mathrm{n}=4)$; treated with amphotericin B $(5 \mathrm{mg} /$ $\mathrm{kg} / \mathrm{d}$ ) via intraperitoneal for 20 days (5 times a week for 4 weeks); and

Control group $(\mathrm{n}=3)$ : infected and untreated.

\section{Treatment of Infected Animals}

The treatment was performed in 2 cycles.

For the first cycle the first lighting system was used and, after the lesion development (approximately 45 days after infection), the 6 groups were treated. In this cycle, contact of $\mathrm{MB}$ with lesions lasted 30 minutes with sequent irradiation with red LED for 30 minutes, performed twice a week for 4 weeks.

After 105 days of infection, Gluc+PDT, PDT and Ampho+PDT groups were submitted to the second cycle of treatment, when the second lighting system was used with more time in contact between $\mathrm{MB}$ and lesion (1 hour) and more irradiation time (1 hour). Groups were irradiated more frequently in this second cycle, 3 times a week for four weeks. In this treatment cycle, no intraperitoneal medication was applied.

\section{Evaluation of Lesion Evolution}

To evaluate the first treatment cycle, lesions were examined every 2 weeks (initiated 30 days after infection and ended after 90 days of infection), to measure the thickness and volume of both hind legs, using the pachymeter (Mitutoyo, Japan) and plestimometer (Ugo Basile S.R.L., Italy) respectively. To evaluate the second treatment cycle, a new check-up was made after 135 days of infection (after the end of second treatment cycle). Thickness and volume of the lesion were calculated by the difference between the infected paw and the noninfected one.

\section{Determination of Parasite Load}

The quantification of parasites in the lesion, the spleen and regional lymph nodes of animals was performed (165 days after infection) according to Buffet et al with modifications. ${ }^{31,32}$ The spleen, the lesion and popliteal lymph nodes of each animal were removed aseptically, weighed and macerated in cell disruptor in 199 culture medium (Gibco, New York, NY, USA) pH 7.2. Suspensions were diluted from $1 / 4$ in the ratio of 2 in cell culture plate with 96 wells. Plates were incubated at $25^{\circ} \mathrm{C}$. Readings were made under an inverted light microscope (Olympus, New York, NY, USA) with 7, 14, 21, 28 days of incubation. The wells were examined searching viable promastigotes, considering a higher dilution in which at least one parasite was found as titer. The experiment was run in duplicates. Parasitic load calculation (number of parasites/gram of tissue) resulted by dividing the geometric media of the reciprocal positive titers and mass of organs $(\mathrm{g})$ multiplied by 53 , which is a fraction of suspension of each inoculated organ in the first well of the plate.

\section{Histological Analysis}

After the end of the second treatment cycle (135 days after infection), euthanasia of the animals was performed by deepening anesthesia. The tissue material collected for histological analysis of injured footpad, spleen, and regional lymph nodes. Tissues were washed with saline solution, sectioned transversally, and immersed in buffered formalin fixative solution at $10 \%$ for 12 hours. After that, tissue was kept in alcoholic solution at $70 \%$ until the standard histological processing at Pathology Laboratory of the State University of Maringá.

The tissues were dehydrated in bathing solutions with increasing alcoholic strength, diaphanized in xylol and included in paraffin. The paraffin blocks were sectioned in rotating semi-automatic microtome (CUT 5062-Slee Medical-Mainz Germany) in fragments of $5 \mu$ m thickness with space of $10 \mu \mathrm{m}$ per cut and adhered to the histological lamina. Four to 5 cuts per lamina were distributed. Hematoxylin-eosin (HE) staining was performed for evaluation of histopathological changes. The analysis of the cuts and the capture of the images were performed with the help of optical light microscope O500R.

The tissue morphology was observed microscopically to study the occurrence of histopathological changes, such as the presence of amastigote nests, polymorphonuclear inflammatory infiltrate and/or mononuclear and tissue necrosis. These parameters were classified as absent (-), rare $(+)$, moderate $(++)$, or intense $(+++)$, according to the frequency of changes. For this, we analyzed a total of 10 microscopic fields for each tissue fragment, and each field was divided into 4 quadrants. The presence of histopathological changes in only 1 of the quadrants was classified as rare $(+)$, in 2 quadrants as moderate $(++)$, and over three quadrants as intense $(+++)$ (Figure 3 ).

\section{Statistical Analysis}

Results were analyzed by Software Bioestat 5.3. The evaluation of normality was performed through Lilliefors test. The values of volume and thickness were analyzed by Student's $t$ test. The analysis of the parasitic load was performed by analysis of variance (ANOVA). Five percent was considered as level of statistical significance.

\section{Results}

At the beginning of the first treatment cycle, forty days after infection, it was noted that the lesions did not present significant differences in relation to their thickness and volume $(P>0.05)$, demonstrating that the groups were not different from each other (Figures 4 and 5).

At the beginning and the end of the first treatment cycle (45 and 75 days after infection, respectively), volume was reduced when comparing the Gluc+PDT, Gluc, Ampho+PDT and Ampho groups with the control group, but this decrease was not statistically significant $(P>0.05)$. At the end of the first treatment cycle, there was no significant difference $(P>0.05)$ of the volume of 


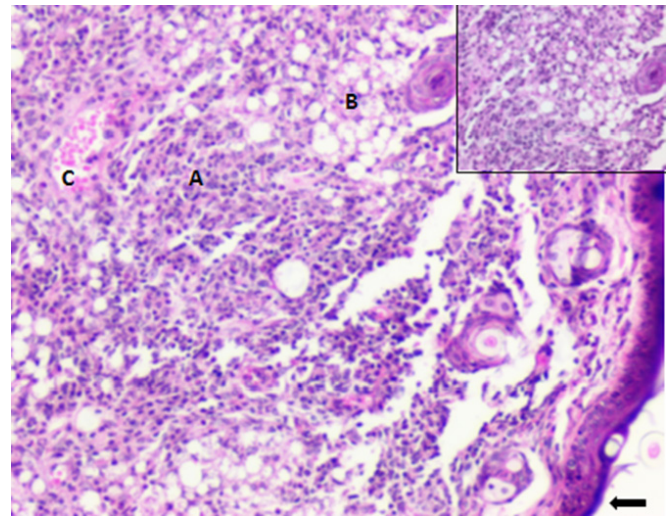

Figure 3. Histological Picture of the Infected Footpad of Mice Infected With Leishmania (Leishmania) amazonensis, in Hematoxylin and Eosin Stain. Reconstituted epidermis after treatment (arrow), mononuclear inflammatory infiltrate (A), amastigote nests (B), and vascular congestion (C) (200x). In the upper left corner photograph in higher magnification (400x) of amastigote nests.

lesions between any of the treated groups in relation to the control group (Figure 4).

Analyzing the footpad volume measurements between the beginning and the end of the second treatment (90 and 135 days after infection, respectively) there was a reduction in volume when comparing the curves of the control group with those of Gluc+PDT, but this difference was not statistically significant $(P>0.05)$. We also noticed an increase in volume in the lesions of the animals of the PDT group in relation to the control group $(P>0.05)$ with 135 days (end of second treatment). None of the treated groups had a significant reduction in lesion volume when compared to the control group $(P>0.05)$ (Figure 4$)$.

In relation to the thickness measurement of the lesion, before and after the first treatment cycle, we noticed a reduction when comparing the control group with the Gluc+PDT and Gluc groups, but that reduction was not statistically significant $(P>0.05)$. In addition, we observed an increase in the thickness of the lesion in the Ampho, Ampho+PDT and PDT groups. At the end of the first treatment, there was no significant reduction of lesion thickness in any of the treated groups when compared to the control group $(P>0.05)$ (Figure 5).

In the second treatment cycle (measured between 90 and 135 days after infection), increased lesion thickness was observed in all groups. After ending this treatment cycle (135 days after the infection), there was no reduction in lesion thickness in any of the treated groups when compared to the control group $(P>0.05)$ (Figure 5).

In the analysis of parasite load quantification in different tissues, it was observed that in the popliteal lymph node there was a statistically significant difference $(P \leq 0.05)$ when we compared the Control group with the Gluc+PDT, Gluc, PDT and Ampho groups, which was not observed when comparing the Control group with the Ampho+PDT group $(P>0.05)$. In the other tissues, spleen and paw with the lesion, the values showed no significant difference in relation to the control group $(P>0.05)$ (Figure 6).

When analyzing histopathological changes of the animals' footpads, we noticed that the group Gluc+PDT presented a smaller number of amastigote nests and lower intensity of mononuclear infiltrate when compared to the Gluc and PDT groups (Table 2 and Figure 7). In the spleen of the animals, we did not observe significant presence of amastigote nests or inflammatory tissue process. No necrosis was observed in any tissue sample. The analysis of regional lymph nodes was not possible due to the

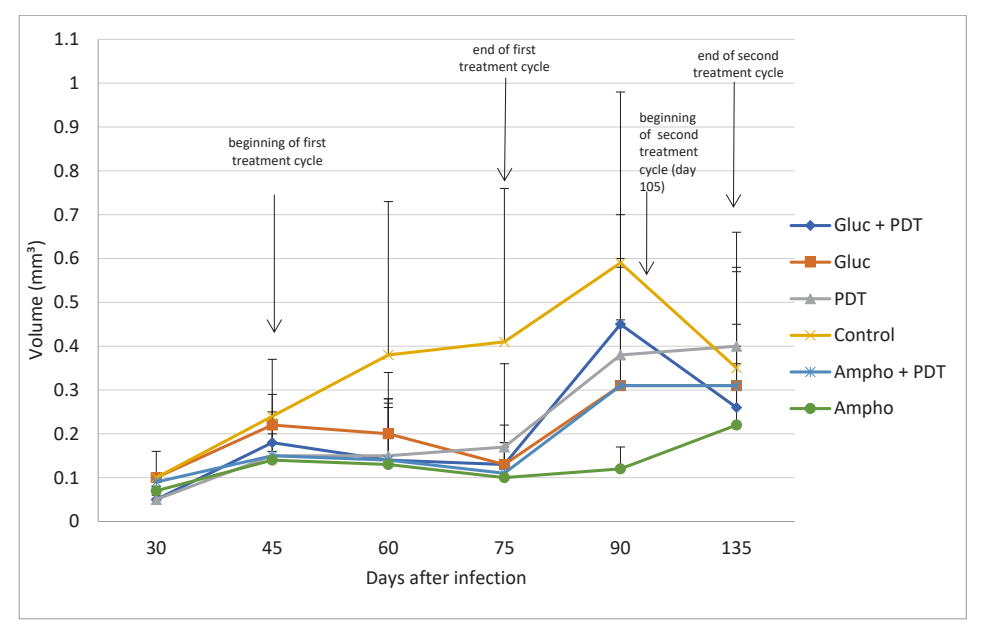

Figure 4. Evolution of the Lesion Volume of the Footpads of Mice Infected With Leishmania (Leishmania) amazonensis. The animals were infected with intradermal inoculation containing 5x105 of promastigotes forms of $L$. amazonensis on the right hind footpad and divided into 6 groups for treatment. Gluc+PDT group $(n=5)$ treated with Glucantime ${ }^{\circledR}(I P)$ and PDT-red LED and methylene blue (MB) in topical aqueous solution, Gluc group $(n=5)$ treated with Glucantime ${ }^{\circledR}(I P)$, PDT group $(n=4)$ treated with PDT-red LED and topical MB, control group $(n=3)$ with non-treated infected animals, Ampho+PDT group $(n=4)$ treated with amphotericin $(I P)$ and PDT - red LED and topical MB and Ampho group $(n=4)$ treated with amphotericin (IP). Measurements were performed using the plestimometer. The volume of lesions was calculated by the difference between the volume of the infected and uninfected footpad and compared to the Control group $(P>0.05)$. 


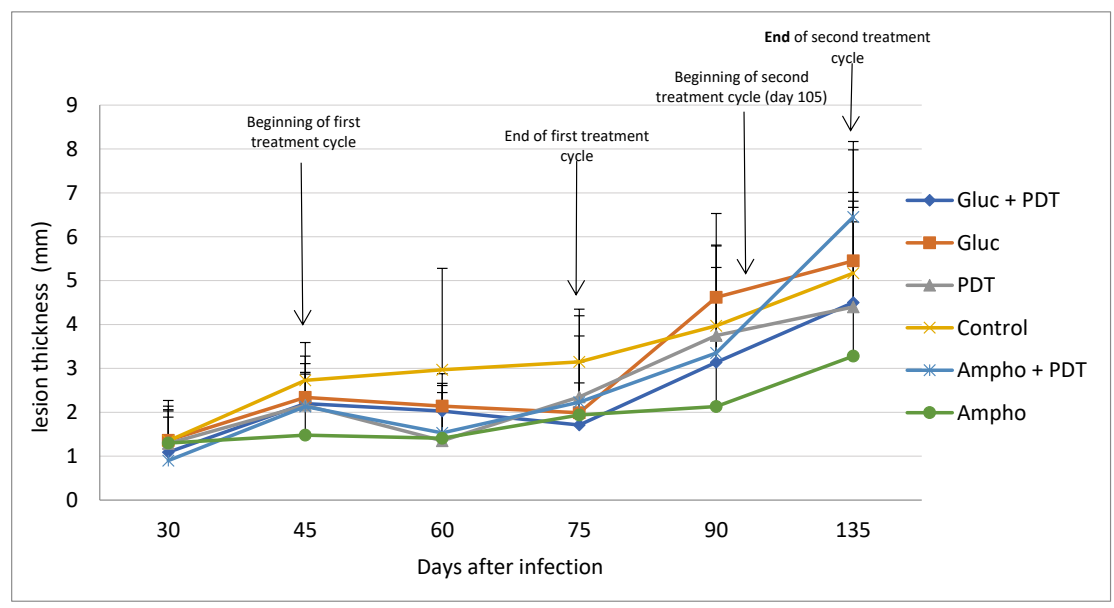

Figure 5. Evolution of Lesion Thickness in the Footpads of Mice Infected With Leishmania (Leishmania) amazonensis. Gluc+PDT group $(n=5)$ treated

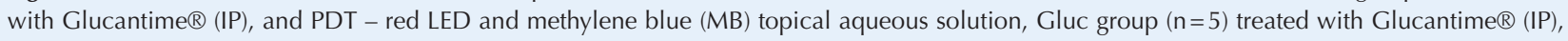
PDT group $(n=4)$ treated with PDT- red LED and topical MB, control group $(n=3)$ with non-treated infected animals, Ampho+PDT group $(n=4)$ treated with amphotericin (IP) and PDT - red LED and topical MB and Ampho group $(n=4)$ treated with amphotericin (IP). Measurements were performed using the pachymeter. Lesion thickness was calculated by the difference between the thickness of the infected and uninfected paws and compared to the control group $(P>0.05)$

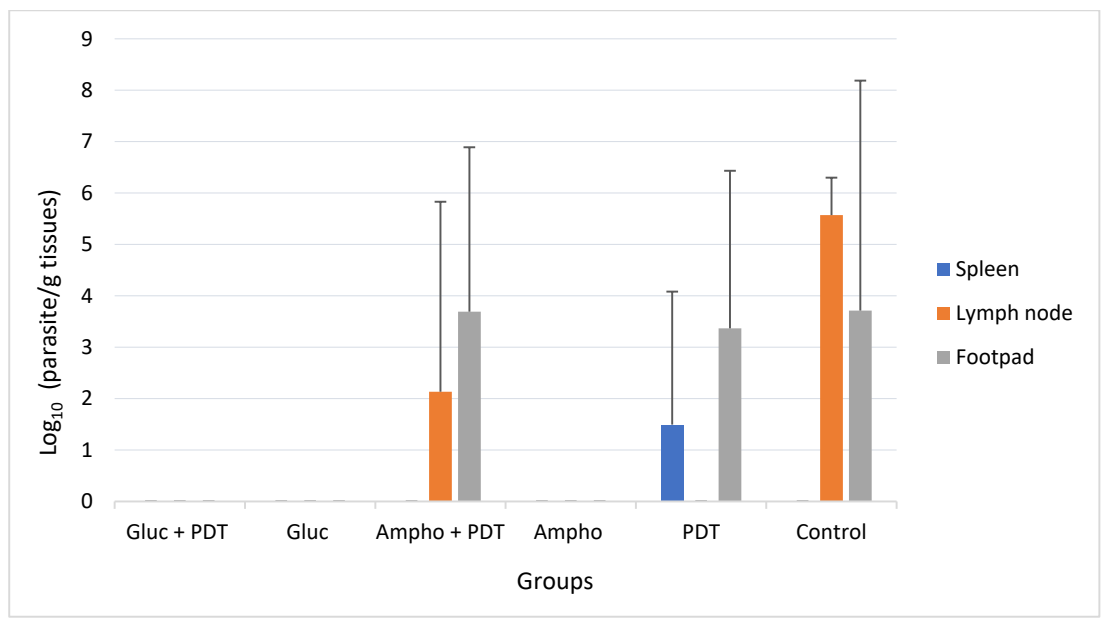

Figure 6. Quantification of Parasite Load on the Spleen, Popliteal Lymph Nodes and Footpad Lesion of mice Infected With Leishmania (Leishmania) amazonensis. Gluc+PDT group $(\mathrm{n}=3)$ treated with Glucantime ${ }^{\circledR}(\mathrm{IP})$ and PDT - red LED and methylene blue in topical aqueous solution, Gluc group $(n=3)$ treated with Glucantime $\mathbb{B}(I P)$, PDT group $(n=3)$ treated with PDT - red LED and topical MB, control group $(n=2)$ with infected and untreated animals, Ampho+PDT group $(n=3)$ treated with amphotericin $(I P)$ and PDT - red LED and topical MB and Ampho group $(n=3)$ treated with amphotericin (IP). Lymph nodes from Gluc+PDT, Gluc, Ampho and PDT groups, when compared to untreated control group $(P<0.05)$.

poor quality of the material after histological processing, making microscopic analysis impossible.

\section{Discussion}

Nowadays, all drugs available in Brazil for the treatment of leishmaniasis are intramuscular and intravenous. The drugs used are the pentavalent antimonials (first choice), amphotericin B and pentamidine (second choice). However, these drugs can cause many side effects, have a long treatment schedule and need an injectable administration. ${ }^{3}$ In view of these therapeutic difficulties, added to drugs resistance, other alternative therapies for cutaneous leishmaniasis have been evaluated, among them PDT. ${ }^{6,14-16}$

Several other studies have demonstrated the benefit of using PDT in the treatment of CL, but a porphyrin precursor (aminolevulinic acid or methylaminolevulinic acid) is the most used photosensitizing agent and the laser as a light source. ${ }^{24-29}$ As those precursors and light source are of high cost and considering that a great number of CL cases occur in underdeveloped countries, dyes like MB have been tested as photosensitizing agents and LED as 
Table 2. Histological analysis of injured footpad, lymph node and spleen of mice infected with Leishmania (Leishmania) amazonensis

\begin{tabular}{lllll}
\hline Groups $^{\text {a }}$ & & Gluc & Gluc+PDT & PDT \\
\hline \multirow{2}{*}{ Amastigote } & Footpads & ++ & + & ++ \\
nests & Lymph nodes & + & + & + \\
& Spleen & - & - & - \\
\hline \multirow{2}{*}{$\begin{array}{l}\text { Mononuclear } \\
\text { infiltrate }\end{array}$} & Footpads & ++ & + & + \\
& Lymph nodes & + & + & + \\
\hline
\end{tabular}

a Gluc+PDT group $(n=3)$ treated with Glucantime ${ }^{\circledR}($ IP) and PDT - red LED and methylene blue in topical aqueous solution; Gluc group $(n=3)$ treated with Glucantime ${ }^{\circledR}(I P) ;$ PDT group $(n=3)$ treated with PDT - red LED and topical MB. The frequency of histopathological changes was graded into absent $=(-)$, rare $=(+)$, moderate $=(++)$ or intense $=(+++)$.

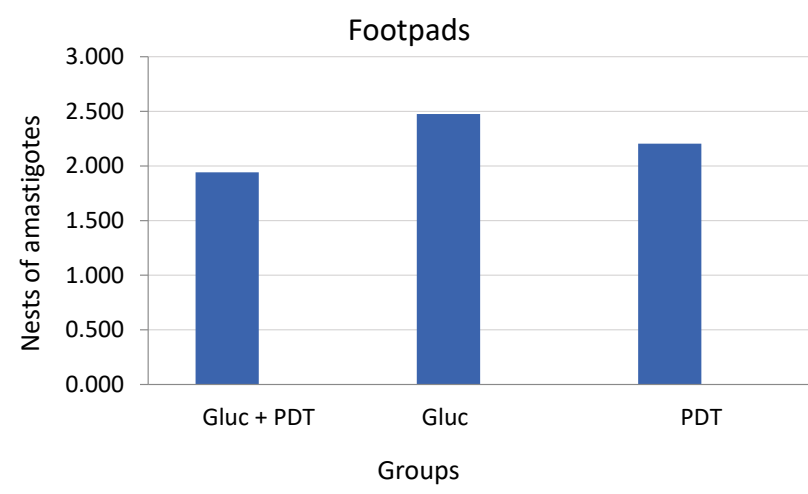

Figure 7. Analysis of Frequency of Amastigote Nests Found on Injured Footpads of Mice Infected With Leishmania (Leishmania) amazonensis. Gluc+PDT group $(n=3)$ treated with Glucantime $\mathbb{}($ IP) and PDT - red LED and methylene blue in topical aqueous solution, Gluc group $(n=3)$ treated with Glucantime ${ }^{\circledR}($ IP), PDT group $(n=3)$ treated with PDT - red LED and topical methylene blue. Frequency of amastigote nests: absent $(-)=$, rare $(+)=1$, moderate $(++)=2$ or intense $(+++)=3$.

light source. Peloi et al and Sbeghen et al utilized topic MB associated with red LED to treat CL, obtaining a decrease in the thickness of the lesion and in the parasitic load. ${ }^{16,30}$ In this study, we evaluated the association of PDT $(\mathrm{MB}+\mathrm{LED})$ with the reference drugs in CL treatment, and observed a reduction in lesion thickness and volume in some of the treated groups, but this difference was not statistically significant. However, there was a significant reduction in the parasitic load in the regional lymph node of the groups Gluc+PDT, Gluc, PDT and Ampho in comparison to the control group, without treatment.

The Gluc+PDT and Ampho+PDT groups, despite having reduced lesion size after treatment (total of 20 IP injections), did not maintain such results after the end of the first therapeutic regimen, since there was a considerable and abrupt increase of the lesion after the end of the first cycle (75 days) and the measurements with 90 days (Figure 5). The doses, number of injections and route of administration used are similar to those used in other studies that have achieved therapeutic success. ${ }^{30,33,34}$
However, the total number of PDT sessions was only 8 in the first cycle of treatment and 12 in the second cycle, which differed from the other experiments, which performed a total of 36 irradiation sessions, in addition to using hamsters and not mice as in this study. ${ }^{16,30}$ Thus, we believe that a longer treatment with PDT may contribute to a greater reduction in the volume and thickness of the lesions.

As observed in Figure 4 and 5, Ampho+PDT group did not present a significant difference in relation to the measures of volume and thickness of the paws when compared to the control group. One hypothesis would be the fact that amphotericin B (organic compound), being an antifungal of the class of the polyenes and possessing a number of double bonds of carbon, can be an excellent target for the singlet oxygen $\left({ }^{1} \mathrm{O}_{2}\right)$ generated in the reaction. The ${ }^{1} \mathrm{O}_{2}$ formed in the PDT is highly oxidizing and attacks double bonds and conjugated rings. This way, it would be consumed in the attack to amphotericin $\mathrm{B}$, and not in the elimination of the protozoa, reducing the therapeutic power of this chemotherapy and, also, of the photodynamic activity, which does not occur with pentavalent antimony (inorganic compound), probably due to its high oxidative power.

The results detected in the PDT group do not confirm the results found in previous studies. ${ }^{16,30}$ This fact may have been influenced by the distance from the light source to the lesion, by the frequency of irradiation and the total number of sessions for the treatment. Peloi et al and Sbeghen et al evaluated the use of PDT in experimentally infected hamsters. The source of irradiation used was located close to the lesion, the irradiation frequency applied was three times a week for a total of three months of treatment with a significant reduction in lesion size. ${ }^{16,30}$ In the present study, two treatment cycles were performed with two different lighting models. In the first one, the LED was used $4 \mathrm{~cm}$ away from the lesion, irradiating for 30 minutes 2 times a week for 4 weeks. In the second, the LED was used $1 \mathrm{~cm}$ away from the lesion and the irradiation was for 1 hour, 3 times a week for 4 weeks. We believe that, after the changes made in the second system, the results were not satisfactory as the lesion was already in advanced evolution. The lighting system used differed from those used in previous works. ${ }^{16,30}$ Since there is no need to anesthetize the animals at each treatment, avoiding stress for the mice and risk of death secondary to anaesthesia use, in this system, the animal is in continuous movement, which may have influenced the angle of incidence of the LED and the total time of light absorption by $\mathrm{MB}$ in the lesion.

The results of the histological evaluation of the infected paw showed that the Gluc+PDT group presented advantages in comparison with the Gluc and PDT groups. The moderate presence of nests of amastigote forms of the parasite and predominance of mononuclear infiltrate, with the absence of polymorphonuclear infiltrate, indicate 
a tendency of cure of the inflammatory process. In the Gluc and PDT groups, we observed an intense presence of mononuclear inflammatory infiltrate, in addition to the intense presence of macrophages filled with vacuoles containing amastigote forms. However, the frequency of amastigote nests in the PDT group differed from that found in the study by Sbeghen et al, which showed only rare amounts of amastigote nests in tissue macrophages in the footpads of hamsters treated with topical MB and PDT. ${ }^{26}$ The statistical evaluation of these data in our study could not be performed because the sample was reduced, since some organs could not be analyzed microscopically due to their poor quality after histological processing.

The results presented show that although there were no significant differences in footpad (thickness and volume) evaluations, there was a tendency of lower growth, as we observed specially in the volume data. Volume measurements can be considered more reliable than those of thickness, as they are less influenced by the degree of ulceration of the lesion. In the evaluation of this parameter, the Gluc+PDT group had lower lesion volumes in comparison to the control group at the end of the first treatment cycle (75 days after infection) and also at the end of the second treatment cycle (135 days after infection). This finding is corroborated by the negativity of the parasitic load in all analyzed organs of the animals of this group.

\section{Conclusion}

These observations, in our point of view, indicate the possibility of using Glucantime ${ }^{\circledR}$ in combination with PDT $(\mathrm{MB}+\mathrm{LED})$ in order to reduce the time of treatment or the dose of medication, which is of first choice in the treatment of CL, with consequent reduction of the risk of side effects. However, new studies should be carried out to evaluate the use of different doses and time of treatment with Glucantime ${ }^{\circledR}$, as well as periodicity, irradiation time, and the total number of PDT sessions with MB to obtain the clinical cure of CL lesions.

\section{Ethical Considerations}

All actions were carried out according to the protocols approved by the Commission on Ethics on the use of animals in experimentation at the State University of Maringá, Maringá, Paraná, Brazil. The study was submitted to the Committee and approved according to report $n^{\circ} 158 / 2013$ of $11 / 17 / 2013$.

\section{Conflict of Interests}

All authors report to have not any funding or business association and conflicts of interest related to this study.

\section{Acknowledgments}

We thank the Coordenação de Aperfeiçoamento de Pessoal de Nível Superior and Fundação Araucária.

\section{References}

1. Leishmaniose. World Health Organization website. http:// www.who.int/mediacentre/factsheets/fs375/es/. Accessed November 21, 2016.

2. Alvar J, Vélez ID, Bern C, et al. Leishmaniasis worldwide and global estimates of its incidence. PLoS One. 2012;7(5): e35671. doi:10.1371/journal.pone.0035671.

3. Brasil. Ministério da Saúde. Secretaria de Vigilância em Saúde. Manual de Vigilância da Leishmaniose Tegumentar Americana. 2nd ed. Brasília: Editora do Ministério da Saúde; 2013. http://bvsms.saude.gov.br/bvs/publicacoes/manual_ vigilancia_leishmaniose_tegumentar_americana_2edicao. pdf.

4. Brasil. Ministério da Saúde. Portal da saúde. Leishmaniose Tegumentar Americana. http://conitec.gov.br/images/ Protocolos/Escopo_PCDT_LeishmanioseTegumentar_ Enquete.pdf. Accessed November 21, 2016.

5. Brasil. Ministério da Saúde do Brasil. Secretaria de Vigilância em Saúde. Manual de Controle da Leishmaniose Tegumentar Americana. 5th ed. Brasília: Editora do Ministério da Saúde; 2000. http://www.saude.pr.gov.br/ arquivos/File/zoonoses_intoxicacoes/leishmaniose/manu_ leishman.pdf.

6. Song D, Lindoso JA, Oyafuso LK, et al. Photodynamic therapy using methylene blue to treat cutaneous leishmaniasis. Photomed Laser Surg. 2011;29(10):711-715. doi:10.1089/pho.2010.2915.

7. Rodrigues AM, Hueb M, Santos TA, Cor JFF. Factors associated with treatment failure of ACL with meglumine antimoniate. Rev Soc Bras Med Tropical. 2006;39(2):139145.

8. Ourives-Neves L, Chrusciak-Talhari A, Gadelha EPN, et al. Estudo clínico randomizado comparando antimoniato de meglumina, pentamidina e anfotericina B para o tratamento da leishmaniose cutânea ocasionada por Leishmania guyanensis. An Bras Dermatol. 2011;86(6):1092-1101. doi:10.1590/S0365-05962011000600005.

9. Ribeiro MN, Pimentel MIF, Schubach AO, et al. Factors associated to adherence to different treatment schemes with meglumine antimoniate in a clinical trial for cutaneous leishmaniasis. Rev Inst Med Trop. 2014;56(4):291-296. doi:10.1590/S0036-46652014000400004.

10. Jeddi F, Caumes E, Thellier M, Jaureguiberry S, Mazier D, Buffet PA. Case report: drug hypersensitivity syndrome induced by meglumine antimoniate. Am J Trop Med Hyg. 2009;80(6):939-940.

11. Oliveira RA, Lima CG, Mota RM, et al. Renal function evaluation in patients with American cutaneous leishmaniasis after specific treatment with pentavalent antimonial. BMC Nephrol. 2012;13:44. doi:10.1186/14712369-13-44.

12. Kashani MN, Firooz A, Eskandari SE, A et al. Evaluation of meglumine antimoniate effects on liver, kidney and pancreas function tests in patients with cutaneous leishmaniasis. Eur J Dermatol. 2007;17(6):513-515. doi:10.1684/ejd.2007.0266.

13. Rojas R, Valderrama L, Valderrama M, Varona MX, Ouellette M, Saravia NG. Resistance to antimony and treatment failure in human Leishmania (Viannia) infection. 
J Infect Dis. 2006;193(10):1375-83. doi:10.1086/503371.

14. Gardlo K, Horska Z, Enk CD, et al. Treatment of cutaneous leishmaniasis by photodynamic therapy. J Am Acad Dermatol. 2003;48:893-896. doi:10.1067/mjd.2003.218.

15. Tardivo JP, Giglio AD, Oliveira CS, et al. Methylene blue in photodynamic therapy: From basic mechanisms to clinical applications. Photodiagnosis Photodyn Ther. 2015;2:175191. doi:10.1186/s12885-017-3179-7.

16. Peloi LS, Biondo CE, Kimura E, et al. Photodynamic therapy for American cutaneous leishmaniasis: the efficacy of methylene blue in hamsters experimentally infected with Leishmania (Leishmania) amazonensis. Exp Parasitol. 2011;128:353-6. doi:10.1016/j.exppara.2011.04.009

17. Bacelar IOL, Tsubone TM, Pavani C, Baptista MS. Photodynamic efficiency: from molecular photochemistry to cell death. Int J Mol Sci. 2015;16(9):20523-59. doi:10.3390/ijms160920523.

18. Machado AE. Terapia fotodinâmica: princípios, potencial de aplicação e prespectivas. Quim Nova. 2000;23:237-243. doi:10.1590/S0100-40422000000200015.

19. Lee Y, Baron ED. Photodynamic therapy: current evidence and applications in dermatology. Semin Cutan Med Surg. 2011;30(4):199-209. doi:10.1016/j.sder.2011.08.001.

20. Stocche RM, Garcia LV, Reis MP, Klamt JG, Evora PRB. Uso do azul de metileno no tratamento de choque anafilático durante anestesia. Relato de caso. Ver Bras Anestesiol. 2004;54(6):809-814. doi:10.1590/S003470942004000600010.

21. Evora PRB, Ribeiro PJF, Vicente WVA, et al. Methylene blue for vasoplegic syndrome treatment in heart surgery: fifteen years of questions, answers, doubts and certainties. Rev Bras Cir Cardiovasc. 2009;24(3):279-288. doi:10.1590/ S0102-76382009000400005.

22. O'Riordan K, Akilov OE, Hasan T. The potencial for photodynamic therapy in the treatment of localized infections. Photodiagnosis Photodyn Ther. 2005;2:247-262. doi:10.1016/S1572-1000(05)00099-2.

23. Van der Snoek EM, Robinson DJ, Van Hellemond JJ, Neumann HAM. A review of photodynamic therapy in cutaneous leishmaniasis. JEADV. 2008:22:918-922. doi:10 $.1111 /$ j.1468-3083.2008.02805.

24. Enk CD, Fritsch C, Jonas F, et al. Treatment of cutaneous leishmaniasis with photodynamic therapy. Arch Dermatol. 2003;139:432-434. doi:10.1001/archderm.139.4.432.

25. Akilov OE, Kosaka S, O'Riordan K, et al. The Role of Photosensitizer molecular charge and structure on the efficacy of photodynamic therapy against Leishmania parasites. Chem Biol. 2006;13:839-847. doi:10.1016/j. chembiol.2006.06.008.

26. Akilov OE, Kosaka S, O’Riordan K, Hasan T. Parasiticidal effect of delta-aminolevulinic acid-based photodynamic therapy for cutaneous leishmaniasis is indirect and mediated through the killing of the host cells. Exp Dermatol. 2007a;16:651-560. doi:10.1111/j.1600-0625.2007.00578.

27. Akilov OE, Kosaka S, O’Riordan K, Hasan T. Photodynamic therapy for cutaneous leishmaniasis: the effectiveness of topical phenothiaziniums in parasite eradication and Th1 immune response stimulation. Photochem Photobiol Sci. 2007b;6:1067-1075. doi:10.1039/b703521g.

28. Asilian A, Davami M. Comparison between the efficacy of photodynamic therapy and topical paromomycin in the treatment of Old World cutaneous leishmaniasis: a placebocontrolled, randomized clinical trial. Clin Exp Dermatol. 2006;31:634-7. doi:10.1111/j.1365-2230.2006.02182.

29. Sohl S, Kauer F, Pasch U, Siman JC, Wetzig T. Photodynamic treatment of cutaneous leishmaniasis. JDDG. 2007;5:12830. doi:10.1111/j.1610-0387.2007.06177.

30. Sbeghen MR, Voltarelli EM, Campois TG, Hernandes L et al. Topical and intradermal efficacy of photodynamic therapy with methylene blue and light-emitting diode in the treatment of cutaneous leishmaniasis caused by Leishmania braziliensis. J Lasers Med Sci. 2015;6(3):101106. doi:10.15171/jlms.2015.03.

31. Buffet PA, Sulahian A, Garin YJ, Nassar N, Derouin F, Culture microtitration: a sensitive method for quantifying Leishmania infantum in tissues of infected mice. Antimicrob Agents and Chemother. 1995;39(9):2167-2168.

32. Lonardoni MVC, Russo M, Jancar S. Essential role of platelet-activating factor in control of Leishmania (Leishmania) amazonensis infection. Infect Immun. 2000;68(11):6355-61.

33. de Mello TFP, Cardoso BM, Lopes SN, et al. Activity of synthetic chalcones in hamsters experimentally infected with Leishmania (Viannia) braziliensis. Parasitol Res. 2015;114(10):3587-3600. doi:10.1007/s00436-015-4581-1.

34. Khalili G, Dobakhti F, Mahmoudzadeh-Niknam H, Khaze V, Partovi F. Immunotherapy with imiquimod increases the efficacy of glucantime therapy of Leishmania major infection. Iran J Immunol. 2011;8(1):45-51.

35. Ganis P, Auitabile G, Mechlinki W, Schaffner CP. Polyene macrolide antibiotic amphotericin B. Crystal structure of the N-iodo-acetyl derivative. J Amer Chem Soc. 1971;93 (18):4560-4564. doi:10.1021/ja00747a037. 